\title{
Búsqueda dinámica y exploratoria: el browsingen los catálogos en línea
}

\author{
J. Carlos Fernandez Molina \\ Eduardo Peis \\ Profesores de la Facultad de Biblioteconomía \\ y Documentación, Universidad de Granada, \\ Colegio Máximo, 18071 Granada, España. \\ Tel: 34-58-243943; Fax: 34-58-243945 \\ E-mail: jcfeman@platon.ugr.es
}

\section{RESUMEN}

$\boldsymbol{L}$ as investigaciones sobre el comportamiento en la búsqueda de información han puesto de manifiesto que se trata de un proceso dinámico e iterativo en el que, además, intervienen elementos de naturaleza subjetiva y afectiva. El reconocimiento de estos factores ha dado lugar a un nuevo modelo teórico de la recuperación de información y, como consecuencia, han aparecido numerosos sistemas que pretenden fomentar la interacción con el usuario. En este contexto, proporcionar métodos para un browsing abierto y exploratorio de la base de datos se convierte en un requisito imprescindible para cualquier catálogo en línea. Atendiendo a estas premisas, se analiza el browsing como comportamiento, su concepto y naturaleza, sus ventajas, sus modalidades y las opciones más adecuadas para organizar la información.

\begin{abstract}
$\boldsymbol{R}$ esearch on information retrieval behaviour has shown that it is a dynamic and iterative process which is also affected by factors of a subjective and affective nature. Recognition of these factors has brought about a new theoretical model of information retrieval and, as a result, numerous systems have appeared intending to encourage user interaction. In this context, to be able to produce searching tools allowing open and exploratory browsing of databases becomes an essential prerequisite in any online catalogue. Taking all this into account, browsing is examined as behaviour and its concept and nature, advantages, modalities and most appropiate ways of organising information are considered.
\end{abstract}

\section{INTRODUCCIÓN}

$\boldsymbol{L}$ a búsqueda de información es una actividad de solución de problemas en la que intervienen varios factores o elementos: un buscador de información, un problema o necesidad informativa, un área de conocimiento, un sistema de búsqueda y un conjunto de resultados, todos y cada uno de los cuales influyen en los demás y en el resultado final del proceso de búsqueda. Sin embargo, tradicionalmente la investigación se ha centrado casi en exclusiva en uno solo de estos elementos: el sistema de búsqueda. Los sistemas de información y los intermediarios que los gestionan han estado regidos por un paradigma — denominado habitualmente bibliográfico- centrado en coleccionar y clasificar textos y en diseñar estrategias para su recuperación.
Bajo este paradigma, la demanda de información es tratada como una concepción única y simple de un problema que permanece invariable a lo largo de todo el proceso de búsqueda y los usuarios son considerados meros receptores de información pasivos e independientes de la situación.

Aunque todos estos presupuestos teóricos son útiles para simplificar la investigación, las búsquedas en la vida real no suelen seguir este patrón. A este respecto, está comprobado que la búsqueda de información, especialmente en sus primeras etapas, está llena de incertidumbre, confusión y ansiedad (Kuhlthau, 1991, 177-209), y que las necesidades de información no suelen ser algo perfectamente definido al principio del proceso de búsqueda, sino que tienen en la reali- dad un carácter dinámico, de manera que van sufriendo modificaciones a medida que el usuario interactúa con el sistema. Las búsquedas reales suelen comenzar simplemente con un término de un tema más amplio o con una referencia relevante, moviéndose a partir de ahí a través de muy diversas fuentes. Cada nueva información que se localiza proporciona nuevas ideas y direcciones para seguir y, por tanto, una nueva concepción de la demanda. En cada etapa no sólo se modifican los términos usados para la equiparación, sino que en realidad es la propia búsqueda la que está cambiando continuamente en todo o en parte, por lo que se denomina búsqueda en desarrollo. Además, en cada etapa, con cada diferente concepción de la búsqueda, el usuario puede identificar información útil y refe- 
rencias, de manera que la demanda se satisface no sólo por el conjunto final recuperado sino por la serie de referencias concretas y trozos de información seleccionados a lo largo de la búsqueda (Saracevic y Kantor, 1988, 197-216).

ste tipo de búsqueda de información es denominado por Marcia Bates (1989, 407-424) como berrypicking, por analogía con la recogida de arándanos, que se va haciendo uno a uno al no encontrarse agrupados en racimos. En su opinión -que compartimos totalmente-, el verdadero modelo de búsqueda de información debe incluir una demanda de información de naturaleza cambiante, en desarrollo, y un proceso de búsqueda que sigue un patrón berrypicking (se recoge poco a poco, en lugar de llevar a un conjunto recuperado único).

De igual forma, este paradigma clásico presupone que todos los usuarios son iguales (Borgman, 1989, 237-251), lo que ha dado lugar a sistemas de recuperación de información que parecen haber sido diseñados para un usuario prototipo, imaginario y anónimo. De esta forma, los usuarios reales se enfrentan al reto de ajustarse a las condiciones preestablecidas por el sistema, lo que provoca que muchos de ellos sólo consigan sacar de él un mínimo partido.

El reconocimiento de todos estos factores inherentes al proceso de búsqueda en sistemas de recuperación de información ha dado lugar a la aparición de un nuevo modelo teórico de la recuperación de información, que tiene en cuenta todos esos elementos de naturaleza subjetiva y afectiva presentes en situaciones reales de búsqueda y recuperación de información por parte de usuarios reales (Dervin y Nilan, 1986, 3-33). Como consecuencia, han surgido los sistemas que intentan fomentar la interacción y el diálogo entre usuario y sistema, lo que supone el reconocimiento del browsing como una estrategia básica e imprescindible para la búsqueda de información, especialmente en sistemas como los catálogos en línea, dirigidos a usuarios finales, cuyas necesidades de información suelen estar poco o mal definidas al principio.

Una vez reconocida y admitida la imperiosa necesidad de que los catálogos en línea proporcionen métodos para un browsing abierto y exploratorio, llega el momento de analizar con atención cuál es su concepto y naturaleza, qué ventajas presenta, en qué consiste como comportamiento, de qué manera se lleva a cabo y cuáles son las opciones más adecuadas para organizar la información de la base de datos.

\section{CONCEPTO Y NATURALEZA}

Aunque el browsing ha sido observado e investigado en el contexto general de la búsqueda de información en la biblioteca y ha asumido cada vez más una parte integral e importante en la interacción hombre-máquina, el concepto y naturaleza del browsing no ha sido estudiado de manera sistemática $\mathrm{y}$, por tanto, no ha sido bien entendido (Chang y Rice, 1993, 231-275).

Una de las excepciones es Marcia Bates (1979, 280-289), que introduce el concepto de tácticas para la búsqueda de información y solución de problemas. Una de las diecisiete idea tactics para encontrar y usar información es la que ella denomina wandering (deambular, recorrer...), definida como el movimiento entre los diferentes recursos informativos, siendo receptivo a las fuentes alternativas y nuevas ideas de búsqueda provocadas por los materiales que nos van apareciendo. Ella resalta la importancia de los descubrimientos o hallazgos fortuitos que pueden hacerse de esta forma, y la influencia de la proximidad física de los ítemes que se examinan.

Otro de los primeros estudiosos y defensores del browsing fue Charles Hildreth (1982, 181-196), quien considera que las personas utilizan este método de búsqueda de información cuando sus criterios de interés no están definidos de manera precisa o están abiertos y sujetos a redefinición.

Más recientemente, David Ellis (1989, 171-212) ha construido un modelo del comportamiento de la búsqueda de información, en el que el browsing aparece como uno de sus seis componentes. En su opinión, se trata de una búsqueda de carácter semidirigido en un área de interés potencial que consta de dos elementos: familiarización y diferenciación. La primera permite al usuario conocer las fuentes de información de esa área, lo que hay disponible; la segunda tiene lugar cuando el usuario desarrolla un conocimiento de las diferencias entre las diversas fuentes, esto es, una apreciación de las diver- gencias entre lo que se encuentra disponible.

La propia Marcia Bates (1989, 407-424), encuadrada en su modelo berrypicking, incluye la técnica de búsqueda denominada area scanning, que consiste en ojear los materiales impresos situados en un área (física o intelectual) donde se han encontrado cosas interesantes con anterioridad. Se utiliza habitualmente con los libros ordenados por el esquema de clasificación bibliotecaria en los estantes de la biblioteca, ya sea siguiendo la ordenación exacta del esquema de clasificación mediante la lectura lineal a lo largo de los estantes, o bien - y más frecuentemente - no siguiendo de manera deliberada ese orden. En la práctica, uno de los aspectos más útiles de este tipo de búsqueda es que se puede consultar visualmente de manera aleatoria el tema de interés tal y como está situado en los estantes, lo que representa una ruptura deliberada del orden de clasificación convencional.

En su opinión, esta técnica constituye la forma más auténtica de browsing en un entorno manual. En este sentido, hay dos cuestiones básicas que deben tomarse en cuenta:

1) El usuario está expuesto a una variedad de áreas relacionadas, algunas de las cuales, dado que se va de aquí para allá, pueden estar relacionadas de manera inesperada, lo que da lugar a descubrimientos o hallazgos fortuitos.

2) El usuario puede mirar directamente en el texto completo de los materiales. Hojeando las páginas y leyendo un pasaje aquí o allá, el usuario consigue una rápida visión global del sentimiento o carácter del autor y de su enfoque o método. Cualquiera que sea ese sentimiento, es prácticamente inaccesible a través de cualquier clasificación o descripción por materias.

El primero de estos dos aspectos, es decir, su papel de pensamiento creativo e innovador ha sido destacado por casi todos los autores que lo han estudiado. Por ejemplo, Apted (1971, 228-230) caracteriza el browsing como una actividad poco clara, poco nítida, que provoca nuevos pensamientos mediante la exposición del usuario a una amplia variedad de estímulos, de manera que el proceso de navegación a través de la información es quizá más valioso en sí mismo que conseguir una respuesta. En una línea simi- 


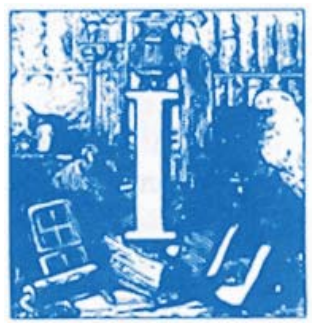

\section{Aunque el browsing ha sido reconocido de manera general por los usuarios como el medio más común para encontrar fuentes de infomación, ha sido una actividad menospreciada en buena parte de la comunidadbibliotecario-documenta lista}

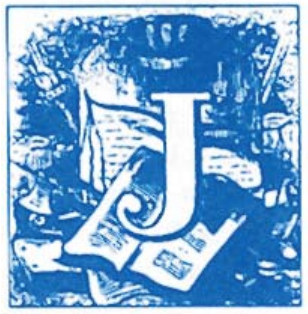

lar, Bawden (1986, 203-216), Davies (1989, 273-301) y O'Connor (1988, 203-210) destacan que el descubrimiento de información durante el proceso de búsqueda crea nuevas estructuras de conocimiento.

La diversidad dentro del concepto de browsing ha sido detectada por todos los investigadores que han estudiado su naturaleza. Utilizando como base la presencia o ausencia de propósito u objetivo o la seriedad o formalidad de la actividad, casi todos ellos coinciden en establecer tres tipos de browsing, aunque con denominaciones diferentes.

Entre ellos se encuentra Herner (1970, 408-415), que propone tres formas de browsing:

a) Dirigido: cuando se tiene en mente un objetivo claro.

b) No dirigido: no existe un objetivo claro y posiblemente sólo se pretende pasar el tiempo.

c) Semidirigido: de carácter intermedio entre los dos anteriores, consiste en el análisis habitual y deliberado de aquellas fuentes que se consideren que probablemente sean útiles. Es el más frecuente en la práctica.

Por su parte, tras definirlo de manera muy pragmática como "el arte de no saber lo que se quiere hasta que se encuentra", Cove y Walsh (1988, 31-37) también desarrollan una taxonomía de tres tipos:

a) Browsing de búsqueda: una actividad fielmente dirigida y estructurada, donde el objetivo deseado es conocido, pero los criterios de búsqueda son imprecisos.

b) Browsing de propósito general: se consultan unas fuentes de información concretas regularmente, ya que es muy probable que contengan ítemes de interés.

c) Browsing de hallazgo fortuito: actividad puramente aleatoria, desestructurada y no dirigida, sin un objetivo consciente.

En cualquier caso, es necesario advertir que durante un mismo proceso de búsqueda es posible pasar de un tipo de browsing a otro (Belkin et al., 1993, 325-344).

El browsing sí se ha distinguido y contrapuesto con cierta claridad a la búsqueda en sentido estricto (querying). Esta distinción resulta bastante evidente en los sistemas de recuperación automatizados, que requieren una clara expresión de la demanda para que una búsqueda se lleve a cabo, de forma que si aquélla no pudiera ser construida, se recurriría al método del browsing. No obstante, esta dicotomía es más aparente que real, ya que no está presente con tanta claridad ni en los sistemas manuales ni en los sistemas automatizados más modernos; quizá sólo resulta diáfana en los primeros sistemas automatizados (por ejemplo, los OPAC de primera y segunda generación) (Hildreth, 1989, 1-24).

\section{VENTAJAS}

Aunque el browsing ha sido reconocido de manera general por los usuarios como el medio más común para encontrar fuentes de información (Baker, 1986, 315-329), ha sido una actividad menospreciada en buena parte de la comunidad bibliotecario-documentalista. Ha habido una fuerte tendencia de los profesionales a considerar que el browsing se utilizaba por los usuarios debido a que no habían sido formados adecuadamente para utilizar sus recursos de información, además de considerar que su existencia era un claro y obvio indicador de las insuficiencias de los métodos de acceso por materias disponibles. El criterio de Donald Urquhart (1976, 7-10) es muy representativo de esta corriente de opinión: browsing es sin duda una actividad útil para un estudiante que no sabe lo que quiere pero debe tener un libro de manera inmediata. Así, se pregunta: ¿es el browsing una actividad sensata en una biblioteca de investigación? ¿es el browsing un superviviente de una antigua costumbre o su supervivencia se debe a la ignorancia o pereza bibliográfica?

Esta tendencia a considerarlo como una parte menor del comportamiento general de búsqueda de información puede tener su origen en que por su propia naturaleza es un comportamiento muy difícil de estudiar formalmente. De hecho, se sabe muy poco acerca de cuestiones básicas tales como cuáles son los factores que lo estimulan, cuál es la tasa de éxito de las diversas formas de browsing o cómo podría definirse lo que constituye "éxito" en este contexto. No obstante, esta situación ha cambiado sustancialmente a partir de la segunda mitad de los ochenta, con trabajos como el de Micheline Han- 
cock (1987, 303-321) o el de David Ellis (1989, 171-212), que han supuesto un importante cambio de tendencia hacia una revaloración de sus ventajas y beneficios como método de búsqueda en un entorno automatizado.

Por tanto, conviene que veamos cuáles son las ventajas concretas que se consiguen con la incorporación de prestaciones de browsing a un sistema automatizado de recuperación de información. Barbara Kwasnik (1992, 53-56) las ha resumido en seis:

1) Browsing no es una actividad pasiva. El usuario hace uso de sus propias estrategias y decide la dirección, ritmo y profundidad de la búsqueda. Esta forma de compartir el proceso entre el usuario y el sistema tiene la ventaja de que hace las búsquedas más dinámicas y flexibles.

2) Las decisiones y estrategias que lleva a cabo el usuario durante el browsing pueden ser utilizadas como un valioso feedback en los sistemas con interfases que lo permiten.

3) El browsing libera al usuario de tener que formular una estrategia de búsqueda precisa, aprovechándose del principio de que es más fácil reconocer lo que es interesante o útil que especificarlo con antelación.

4) Si el sistema proporciona un espacio bien conectado y ayudas navegacionales, mediante browsing es posible explorar la información de un sistema sin tener conocimiento previo de su contenido. De esta forma, en este tipo de sistemas puede ser inapreciable para los usuarios el introducirse en áreas temáticas nuevas o desconocidas.

5) El usuario tiene la oportunidad de generar nuevos puntos de vista con nuevas e insospechadas combinaciones o agrupaciones de información.

6) Un entorno "browsable" aprovecha el hecho de que la información se presente en su contexto. Éste ayuda a reducir la ambigüedad y además proporciona información que ya no necesita ser suministrada expresamente por el diseñador del sistema en alguna otra forma.

En el contexto específico de los OPAC, y tras analizar tres interfases de OPAC comerciales, John Akeroyd (1990, 33-52) señala cuatro razones por las que los usuarios utilizan el browsing en sus búsquedas de información: a) para corregir errores de la introducción (por ejemplo, haciendo browsing hacia atrás hasta la posición correcta); b) para establecer el alcance y contenido de la terminología (haciendo browsing en los diversos índices de materia); c) para expandir el ámbito de los documentos recuperados (por ejemplo, haciendo browsing en una lista clasificada jerárquica); y d) para especificar un subconjunto de un conjunto recuperado en una búsqueda booleana.

David Bawden (1993, 71-85), por su parte, considera que son dos sus ventajas únicas y específicas:

a) La capacidad para encontrar analogías, conexiones y nuevas líneas de pensamiento, esto es, para estimular el uso creativo e innovador de las fuentes de información.

b) Constituye una forma simple y amigable de acceso a las fuentes de información, en especial para usuarios inexpertos y ocasionales.

En el contexto de los OPAC, esta segunda ventaja es sin duda la más significativa, ya que permite reducir al mínimo la necesidad de formular una demanda bien expresada y de utilizar los elementos más sofisticados y complicados del lenguaje de interrogación, desde el momento en que cualquier forma de browsing se basa en un acceso inicial muy simple a la fuente de información, un análisis de ítemes y alguna forma de seguimiento igualmente sencilla.

\section{EL COMPORTAMIENTO DE BROWSING}

Aunque el browsing es uno de esos comportamientos intuitivamente simples que todos usamos, resulta bastante difícil definirlo con una adecuada precisión para incorporarlo al diseño de sistemas de información. La noción de browsing se toma a menudo como un comportamiento evidente por sí mismo, como algo obvio, pero no hay acuerdo acerca de su definición o de su interacción con otros aspectos de uso del sistema. Por tanto, es necesario descubrir qué hacen realmente los usuarios y qué consiguen funcionalmente cuando efectúan browsing.

Con este objetivo en mente, Barbara Kwasnik (1992, 53-56) ha cónfigurado un modelo descriptivo que especifica cuáles son las funciones típicas del brow- sing como método de búsqueda de información, que además puede servir de base para el diseño de sistemas de información. En su estudio, Kwasnik considera que el browsing es fundamentalmente un movimiento en un espacio conectado. Con objeto de conseguir este movimiento, las personas llevan a cabo determinadas acciones: cambian su mirada, alteran su posición, pasan por alto cosas, echan un vistazo a determinadas cosas, retroceden, se paran o hacen una pausa y responden a fenómenos que les resultan interesantes. Una función de browsing estaría constituida por un conjunto o grupo de algunas de estas actividades. Tras estudiar una serie de casos, ella ha identificado una lista de funciones a las que se recurre con regularidad:

a) Escoger un lugar de partida: el usuario se sitúa en una posición que le permita alcanzar buenos resultados.

b) Orientación: se aprende la estructura y contenido del entorno. Esto no sucede una sola vez y al principio, sino que se desarrolla y modifica a medida que progresa la actividad.

c) Marcar el lugar: marcar una posición para una potencial consideración posterior. Los indicadores de lugar físicos (por ej. poner el dedo entre unas páginas) o mentales (memorizar una marca o señal) son experimentales y pueden ser objeto de cambio cuando nuevas experiencias modifiquen $\mathrm{o}$ reestructuren los objetivos e intereses.

d) Identificación: el proceso de browsing se basa en el reconocimiento de ítemes potencialmente interesantes o definitivamente no interesantes. La decisión de seguir más adelante o parar depende de la habilidad de la persona para resumir el contenido probable de una posición concreta.

e) Resolución de anomalías: las anomalías, es decir, cosas que son confusas, poco claras o que no parecen adecuadas, ocurren tanto en la estructura como en el contenido del entorno de browsing. El importante esfuerzo para resolverlas, incluso cuando el ítem que está siendo resuelto no parece ser de gran interés, está en evidente consonancia con el hecho de que los usuarios crean estructuras y se orientan a sí mismos a medida que avanzan. 
f) Comparación: los usuarios hacen comparaciones a todos los niveles: comparan un ítem con otro, el entorno como un todo con otros entornos, y diversos aspectos de la estructura del entorno de browsing. Estas comparaciones sirven para orientar, identificar y solidificar los propósitos y objetivos.

g) Transiciones: son los movimientos o pasos desde una posición a otra, porque se ha conseguido suficiente información, porque no interesa, etcétera.

Todas estas acciones hacen referencia tanto al browsing llevado a cabo en un entorno manual como en uno automatizado. Sin embargo, hay algunas diferencias que es necesario comentar. Así, mientras este último es básicamente cognitivo, el primero tiene un mayor componente físico (andar, girar la cabeza, tocar...). A este respecto, diversos autores han considerado que el browsing queda desvirtuado si no se lleva a cabo en un entorno manual (Norton, 1984, 317-323). El origen de estas actitudes se encuentra en las importantes limitaciones que presentaban los primeros sistemas de información automatizados, cuya flexibilidad y capacidad para la interacción eran muy escasas. De hecho, hay que reconocer que los sistemas automatizados todavía no han conseguido proporcionar algunas de las características intrínsecas del browsing. Por ejemplo, Marcia Bates (1989, 407-424) señala que una de las ventajas del browsing consiste en leer el texto de los documentos no de manera secuencial, sino hojeando o saltando de un lugar a otro de ellos hasta conseguir una impresión o sensación general. Sin embargo, todavía no es habitual que los OPAC provean acceso al texto completo de los documentos, sino únicamente a sus sustitutos (resúmenes, términos de indización, títulos, etcétera). Otra de las características esenciales del browsing es el movimiento físico entre la colección. Para proporcionarlo se han diseñado sistemas que reproducen mediante interfases gráficos de usuario, el entorno espacial de una biblioteca. Las salas, estantes, libros y servicios son reproducidos mediante la simulación de un entorno tridimensional, donde los usuarios pueden interactuar con las colecciones de documentos como si lo estuvieran haciendo en una biblioteca física. Este tipo de sistemas experimentales son cada vez más numerosos, por lo que vale la pena que comentemos algunos de ellos. Uno de los pioneros fue el diseñado por Benest et al. $(1987,905910)$ en el departamento de informática de la Universidad de York. En 61, lo primero que se ofrece al usuario es una representación de los índices (materia, autor, kwic, signatura, etcétera), cada uno de los cuales se simboliza como un libro en un estante que el usuario puede seleccionar con el ratón. El índice escogido se presenta en la pantalla como un libro abierto con dos páginas a la vez, y el usuario puede hojear estas páginas de entradas del índice alfabéticamente, con la posibilidad de saltar a una letra concreta si se desea. Una vez que se selecciona la entrada del índice, el libro es localizado físicamente sobre un estante simulado. Los tamaños físicos de los libros están en relación con el número de páginas, formato, etcétera. El usuario puede hacer browsing a través de las obras clasificadas en el estante, y es posible hojear las páginas, saltando de una a otra, poniendo marcas en ellas, etcétera, de la obra seleccionada.

Más recientes son los diseñados por Beheshti (1992, 220-228), que permiten al usuario moverse entre los recursos del catálogo de la misma forma en que lo haría entre los fondos de una biblioteca, y el Science Library Catalog desarrollado en la Universidad de California en Los Angeles (UCLA). La interfase de usuario de este catálogo experimental, dirigido específicamente a los niños, utiliza la jerarquía de la Dewey Decimal Classification como una metáfora de las estanterías de la biblioteca, con objeto de ajustarse al modelo mental de los niños respecto al catálogo bibliotecario y la biblioteca misma. Para hacer browsing el niño se mueve a través de las estanterías seleccionando categorías apuntando y pulsando con el ratón, que es el único dispositivo de introducción. Para moverse en la jerarquía sólo es necesario pulsar en la estantería anterior expuesta a la izquierda. En el nivel más bajo de cada rama de la jerarquía arbórea se encuentran los registros de los libros, que se seleccionan simplemente pulsando en su título. Los datos de los registros aparecen en la pantalla en la posición típica que ocupan en un libro, es decir, en la portada, contraportada y página siguiente. Por otro lado, si se escoge la opción library map aparece una imagen con la distribución física de la biblioteca (Borgman et al., 1995, 663-684).

\section{FORMAS DE BROWSING}

De acuerdo con David Bawden (1993, 71-85), es posible distinguir tres formas básicas de browsing. La primera consiste en encontrar ítemes similares a uno o más ya conocidos cuando la naturaleza de la similitud está definida sólo de manera imprecisa. La segunda estriba en seguir una categorización predefinida -probablemente jerárquica-a través de un espacio de información para identificar ítemes interesantes. Y, por último, la tercera implica la obtención de una visión general de la diversidad de los ítemes de que consta un espacio de información para identificar sus áreas interesantes.

Vamos a usar este concepto tripartito de browsing como punto de partida para analizar con algo más de detalle cómo se ha aplicado en el contexto bibliotecario, incluyendo algunos ejemplos concretos de sistemas que han adoptado alguna de estas tres formas de browsing.

\section{Guiado por estructura}

El método que se ha utilizado más habitualmente en una biblioteca es el segundo de los establecidos por Bawden: las acciones de browsing se llevan a cabo a través de una categorización impuesta por la biblioteca, normalmente un esquema de clasificación para ordenar los libros en los estantes o una serie de índices fáciles de usar.

No obstante, la estructura o guía que se ofrece más frecuentemente en los sistemas automatizados para ser objeto de browsing es la lista alfabética (de nombres de autores, encabezamientos de materia, palabras del título, etcétera), ya sea desde el principio o a partir del punto que el usuario ha seleccionado en su búsqueda. Evidentemente, estas listas pueden ser recorridas arriba y abajo hasta encontrar algo que nos interese, pero la premisa en la que se basan — las palabras con la misma raíz tienen significados similares — tiene una validez muy limitada.

Casi la totalidad de los sistemas actuales que permiten el browsing tiene el examen de listas alfabéticas como principal 
método de acceso. No obstante, para mejorar sus prestaciones las combinan con una buena interrelación de los atributos de los ítemes, aprovechándose de los múltiples puntos de acceso que proporcionan. Este método era el seguido por los primeros sistemas experimentales como THOMAS (Oddy, 1977, 1-14) o BROWSE (Palay y Fox, 1981, 310-324) y por el primer sistema comercial realmente operativo, TINlib (Noerr y Bivins-Noerr, 1985, 205-213). La interrelación de los atributos de los ítemes permitía llevar a cabo acciones tales como examinar una lista de encabezamientos de materia, escoger uno de interés, examinar los registros que le corresponden, seleccionar uno de ellos, usar su número de clasificación para buscar otros de la misma materia, etcétera.

Un enfoque algo distinto es el seguido en el sistema experimental $\mathrm{I}^{3} \mathrm{R}$ de Croft $\mathrm{y}$ Thompson (1987, 389-404). Uno de los sistemas expertos que lo componen es el denominado Browsing Expert, que gestiona una función de browsing que permite al usuario comenzar su búsqueda por cualquier documento, autor o término en la base de conocimiento y seguir las conexiones hasta cualquier otro documento, autor o término de la base. Ésta se muestra gráficamente al usuario como una red de nodos y enlaces: los nodos representan a las entidades (documentos, autores, términos) y los enlaces a las relaciones (proximidad máxima, documento citado, documento citante, autores y términos) entre las entidades.

Otros dos posibles tipos de estructura que se pueden utilizar como medio para browsing son los tesauros y los esquemas de clasificación bibliotecaria. La primera posibilidad ha sido planteada por David Ellis (1989, 171-212) o por Richard Pollard (1993, 345-357), entre otros, pero, dada su gran amplitud temática, no parece muy apropiada para un catálogo en línea. La segunda, que sigue el método usado habitualmente para ojear las colecciones impresas de las bibliotecas, ha sido propuesta por numerosos autores en muy diversos experimentos. En nuestra opinión, esta vía puede conducir a resultados satisfactorios, por lo que le vamos a dedicar una atención especial.

El sistema pionero en utilizar un esquema de clasificación para el acceso y el browsing por materias en línea fue el diseñado por Freeman y Atherton (1968, 122-152). Crearon un fichero con los nú- meros de ciencia nuclear y las descripciones en lengua inglesa correspondientes a esos números de CDU, incluyendo también las referencias cruzadas y las notas de alcance de las tablas. Por otro lado, cargaron un fichero bibliográfico en el que los números de $\mathrm{CDU}$ formaban parte del registro de índice de los ítemes bibliográficos. Para la búsqueda y recuperación se usó un sistema interactivo denominado AUDACIOUS, basado en una de las primeras versiones del Recon de la NASA. Los usuarios introducían sus ecuaciones de búsqueda expresadas mediante términos o frases en lenguaje natural o mediante un nú-mero de CDU. El sistema permitía la conexión entre los términos utilizados y los números de CDU, situaba la búsqueda en su contexto jerárquico mostrando los números anteriores y posteriores al escogido con su correspondiente texto, permitía el uso de los operadores booleanos, etcétera.

Ya mucho más recientemente nos encontramos con el ya comentado Science $\mathrm{Li}$ brary Catalog y el Dewey Online Retrieval System (DORS), ambos desarrollados en la Universidad de California en Los Angeles (UCLA). Los responsables de este último, Liu y Svenonius (1991, 359-375), señalan las ventajas que ofrece la estructura típica de un sistema de clasificación, que agrupa el vocabulario asociado con un concepto a diferentes niveles de especificidad. En comparación con el tesauro, consideran que aunque éste ejerce un tipo de control de vocabulario similar, un sistema de clasificación va más allá porque estructura semánticamente no sólo el vocabulario asociado con conceptos sino también los .,conceptos mismos. En su opinión, las clasificaciones pueden ser vistas como redes semánticas o como árboles de conocimiento, por lo que tienen más en común con una base de conocimiento que un tesauro. Siguiendo con su argumentación, consideran que estas características estructurales de los sistemas de clasificación pueden ser aprovechadas en estrategias de búsqueda en línea muy diversas, pero que la principal de ellas es sin duda el browsing.

La característica más destacable de este sistema es que genera índices en cadena de manera totalmente automática. Para su creación se extrajeron términos significativos del índice relativo y de las cabeceras de las tablas de la DDC, que se constituyeron en cadenas basándose en sus relaciones jerárquicas. Un encabezamiento de este índice tiene la forma de $X: Y: Z$, donde $X$ es el término "foco", e Y y Z son términos contextuales que suministran el contexto temático para el término "foco". Así, Y es una clase más general que $\mathrm{X}, \mathrm{y} \mathrm{Z}$ es una clase más general que $\mathrm{Y}$.

DORS proporciona buenas prestaciones para un browsing global, ya que da dos visiones de la base de conocimiento representada por la DDC: una presentación del índice en cadena y otra de las tablas. La primera es una presentación en forma de cadena que contextualiza los términos de búsqueda en todas las jerarquías en las que aparecen, mostrando todas las perspectivas o puntos de vista desde los que se trata el tema designado por los términos. La segunda es una presentación en forma de árbol que proporciona un método de abajo hacia arriba para el browsing, en cada paso se muestra al usuario la secuencia completa de clases bajo la clase designada por los términos de búsqueda. Mientras la presentación del índice en cadena facilita el browsing global a través de las jerarquías, la presentación de las tablas facilita un browsing en profundidad dentro de las jerarquías.

\section{Equiparación por similitud}

Los defectos de los sistemas de recuperación basados en la lógica booleana y la equiparación exacta han dado lugar a la aparición de los sistemas basados en la similitud y la equiparación parcial, que tienen ventajas tales como que nunca dan resultado nulo, que ayudan en el análisis de los resultados de la búsqueda, ya que los ordena de acuerdo con su relevancia, etcétera.

Estas técnicas han probado su eficacia suficientemente, por lo que parece probable que todos los sistemas de recuperación se basarán en ellas en un futuro próximo; sin embargo, su aplicación a sistemas de recuperación de información bibliográfica con prestaciones de browsing es muy escasa todavía. Sólo se ha implementado en algunos sistemas experimentales de los que el más significativo es INSTRUCT. Éste es un sistema que ha sido diseñado como herramienta para experimentar con las técnicas avanzadas de recuperación y para mostrarlas a los alumnos de la Universidad de Sheffield. Una vez identificados uno o varios documentos relevantes, INSTRUCT permite 
dos tipos de browsing: búsqueda en cadena y búsqueda "semilla". A partir de un documento considerado relevante se inicia una búsqueda en cadena que va llevando al usuario hacia los documentos relacionados, para lo que el sistema utiliza rutinas estadísticas de búsqueda de tipo best match y mecanismos de clustering que sitúan juntos a los documentos relacionados. Por su parte, el segundo tipo es una búsqueda best match en la que los términos de la demanda original son sustituidos por los términos —reducidos a la raíz- extraídos del título y resumen del documento considerado como relevante (que constituyen la denominada "semilla"). El resultado de la búsqueda es una lista de documentos ordenados de acuerdo con su similitud decreciente respecto a la "semilla" (Wade y Willett, 1988, 44-61).

Las investigaciones llevadas a cabo en INSTRUCT han servido como base para diseñar SIBRIS, un sistema de recuperación con amplias prestaciones de browsing desarrollado por Pfizer Central Research, una institución británica de investigación farmacéutica (Wade et al., 1989, 249-260).

\section{De visión global}

Esta tercera forma de browsing está mucho menos desarrollada que las anteriores, aunque cada vez está más clara la importancia de proporcionar una visión general del espacio de información en el contexto de los sistemas hipertexto para evitar sus problemas de desorientación.

Hay un interesante antecedente que se implantó en las bibliotecas norteamericanas entre los años 30 y 50 y que se denominó browsing room. Consistía en poner a disposición de los usuarios un subconjunto de la colección de la biblioteca - que se cambiaba con frecuencia- en una sala aparte, libre de algunas de las normas bibliotecarias (respecto a fumar, a hablar en voz alta, etcétera). La elección de la colección de browsing se hacía de manera que promoviera la lectura espontánea y mostrara la cobertura o variedad de la colección de la biblioteca (Apted, 1971, 228-230). Desgraciadamente, esta idea desapareció y no se ha implementado nada parecido en los catá- logos automatizados, ni siquiera a nivel experimental.

Uno de los escasos sistemas que proporcionan esta forma de browsing es EYE-BROWS, que genera una visión global de un documento por el sencillo método de escoger frases con una alta proporción de palabras significativas, es decir, aquellas que tienen las frecuencias más altas —eliminando las palabras vacías-dentro del documento. El objetivo de esta prestación de EYEBROWS era describir el contenido del texto y dar una idea de su estructura y temática como punto de partida para el browsing (Cove y Walsh, 1988, 31-37).

\section{Organización de la información}

Para que una fuente de información sea realmente útil para llevar a cabo actividades de browsing es necesario que sea organizada de manera apropiada, esto es, la forma en que los recursos informativos estén organizados o estructurados tiene una importante influencia en el tipo y facilidad de browsing (Chang y Rice, 1993, 231-275; Ellis, 1989, 171-212 ).

Por otro lado, dado que se trata de una actividad de naturaleza muy personal, condicionada por los estados de conocimiento de los usuarios, cualquiera que sea la forma de organización adoptada, debe ser una que anime y apoye que aquéllos hagan browsing por sí mismos. En opinión de David Bawden (1993, 71-85), la información puede ser organizada en una de las dos formas siguientes: a) de una forma semialeatoria, permitiendo la conexión entre áreas temáticas; y b) mediante un completo procesamiento, específico para las necesidades de browsing. En este punto nos encontramos con una antigua polémica: ¿es preferible proporcionar una amplia colección para el browsing o presentar la colección dividida en pequeñas áreas temáticas? La segunda de estas dos opciones fue fuertemente defendida por Philip Morse (1970, 391-408) en un contexto de browsing en colecciones impresas, al considerar que era más conveniente ofrecer subcolecciones de pequeño tamaño que pueden ser hojeadas en 15 ó 30 minutos. Más recientemente, y ya en el contexto de los sistemas automatizados, Charles Hil- dreth (1982, 181-196) también defiende esta postura. Los actuales problemas de sobrecarga de información nos permiten afirmar que esta segunda opción es la acertada.

En opinión del propio Hildreth, la estructura de los OPAC resulta bastante adecuada para soportar el browsing, ya que proporciona una buena cantidad de información interrelacionada (autores, títulos, códigos de clasificación...) que permite al usuario conseguir el acceso a material relevante. No obstante, también se dio cuenta de que la mayoría de los catálogos carecía de las adecuadas prestaciones de presentación para que el usuario pudiera ver las conexiones entre registros y atributos y pudiera valorar si un determinado ítem era relevante o no. Por desgracia, la situación descrita entonces por Hildreth no ha sufrido cambios demasiado significativos.

Una buena muestra del segundo tipo de organización establecido por Bawden es el sistema TINlib. Las múltiples conexiones que establece entre ítemes y atributos permiten al usuario navegar con mucha facilidad a través de la estructura de la base de datos. Para conseguir esta capacidad es necesaria una compleja estructuración y configuración de la base de datos (Noerr y Bivins-Noerr, 1985, 205-213).

Principios similares seguía el sistema experimental BROWSE (Palay y Fox, 1981, 310-324), capaz de tratar con las preguntas clásicas que un usuario se plantea cuando hace browsing en una biblioteca:

a) Si el libro $X$ es interesante, ¿qué más hay del autor que lo ha escrito?

b) Si el libro $X$ es interesante, ¿qué otros libros tienen la misma categoría o son del mismo tipo?

c) Si una comunicación de un congreso es interesante, ¿qué más comunicaciones o ponencias se han presentado en ese congreso?

d) $\mathrm{Si}$ el autor de un artículo interesante pertenece a la institución X, ¿qué más se ha publicado en esa institución?

e) Si hay un artículo interesante en una revista, ¿qué más aparece en esa revista?

El sistema proporciona respuesta a este tipo de cuestiones dando al usuario vías fáciles y rápidas de acceso entre los registros relacionados. Además del método de las listas alfabéticas, utilizado para 
examinar los valores de los atributos concretos (nombres, palabras clave, etcétera), proporciona una clasificación jerárquica que permite al usuario navegar hasta los atributos que desea ver presentados. Para soportar todas estas prestaciones la base de datos tiene una compleja estructura en forma de red que puede verse en pantalla.

En la actualidad, parece claro que el tipo de estructura más completo y desarrollado para proporcionar buenas prestaciones de browsing es el hipertexto. La idea básica del hipertexto es muy simple: toda la información se encuentra conectada en un conjunto textual que está compuesto por una serie de nodos - que suele representar un ítem o un término-y de enlaces - que representan las conexiones asociativas entre los nodos- De esta forma, se va estableciendo una compleja red interconectada que puede ser recorrida con facilidad. La idea del sistema bibliotecario basado en el hipertexto fue propuesta por Hjerppe (1986, 211-232) como una extensión y mejora del catálogo tradicional, utilizando para ello el material ya disponible en los registros bibliográficos. En su opinión, las características distintivas de un "hipercatálogo" en relación con el catálogo clásico son las siguientes (Hjerppe, 1989, 177-209):

El browsing y la navegación son los * Modos básicos de usar el catálogo,

Aunque también proporciona los métodos de búsqueda tradicionales.

* Su grado de estructura interna es mucho mayor.

* Dispone de medios alternativos para presentar e ilustrar la información, las estructuras y las relaciones.

* Tiene herramientas para establecer relaciones y seguir direcciones o senderos.

* Es dinámico por naturaleza, no sólo por el crecimiento del número de registros.

Cuenta con modelos de usuarios, tanto estereotipados como individualizados, para facilitar la adaptación a los diferentes tipos de usuarios así como a las necesidades individuales.

* Contiene mayor cantidad y diferente información que el catálogo tradicional, en especial en términos de conexiones y relaciones entre campos, registros y ficheros.

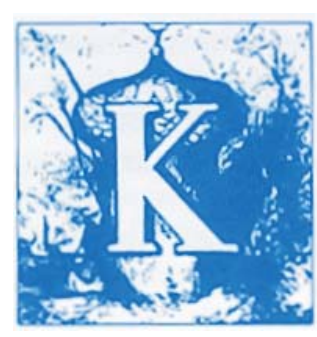

\section{En la actualidad, parece claro que el tipo de estructura más completo y desarrollado para proporcionar buenas prestaciones de browsing es el hipertexto}

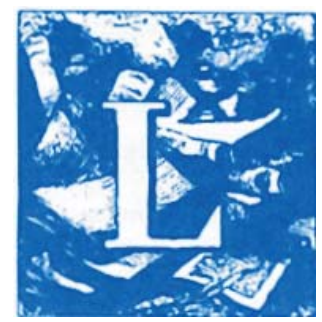

* Proporciona información no sólo sobre ftemes individuales, sino también sobre colecciones.

El principal inconveniente que presentan es el de la (des)orientación (Edwards y Hardman, 1989, 105-125; Foss, 1989, 407-418), que se agrava en bases de datos de gran tamaño donde la estructura de nodos y enlaces puede llegar a ser demasiado grande y compleja, haciendo que la navegación a través de ella sea difícil y tediosa.

Para resolver esto se han propuesto varias soluciones. Una de ellas consiste en el uso de técnicas de inferencia para permitir que el sistema identifique nodos relacionados con la demanda del usuario, de manera que el sistema produce una lista de nodos candidatos que puede ser usada como punto de partida para la navegación del usuario (Turtle y Croft, 1991, 187-222). Mucho más interesante nos parece la opción de mejorar el espacio informativo con una serie de pistas estructurales que permitan al usuario identificar los nodos relevantes (Pollard, 1993, 345-357), por lo que la vamos a comentar con más detalle.

Las pistas estructurales, cuyo objetivo es el de ayudar a los usuarios a formar un modelo mental del espacio informativo que les facilite la navegación, pueden proporcionarse por varios métodos. Uno de los más usados es un browser gráfico, que suele presentar un diagrama esquemático del hipertexto en el que los nodos se representan como cajas etiquetadas y las conexiones entre los nodos como líneas, de manera que se muestra al usuario cómo es el espacio informativo global y cómo está conectado, por lo que constituyen un buen medio para moverse de un nodo de información a otro. No obstante, puede haber problemas si se generan mapas demasiado grandes y confusos, para lo que puede ser una buena solución la existencia de una representación a varias escalas entre las que sea sencillo moverse (Dillon, 1992, 22-25).

Otro método para proporcionar pistas estructurales es usar una arquitectura de dos niveles en la que la colección documental es complementada por una colección auxiliar de datos, siendo esta última la que se ojea con objeto de identificar los términos de interés. Una vez localizados, es posible examinar los documentos asociados con tales términos de la colección 
auxiliar de datos para evaluar su relevancia en relación con la necesidad de información del usuario. En nuestra opinión, una buena forma de construir esa colección auxiliar de datos sería el uso combinado de técnicas de clustering y esquemas de clasificación bibliotecaria, que permitirían agrupar los términos de acuerdo con su especificidad en cada una de las áreas temáticas. Este método ha sido seguido en los dos catálogos en línea experimentales que consideramos más prometedores: el ya mencionado Science Library Catalog (Rosenberg y Borgman, 1992, 171-184) y el denominado Cheshi$r e$, desarrollado en la Universidad de Berkeley (Larson, 1991, 133-173; Larson et al., 1996, 555-567).

\section{CONCLUSIONES}

Las investigaciones sobre el comportamiento en la búsqueda de información han puesto claramente de manifiesto que se trata de un proceso dinámico e iterativo, no determinista. De hecho, incluso el usuario que sabe con precisión lo que quiere y usa el catálogo simplemente para localizar un ítem concreto y determinar su disponibilidad, puede escoger varias formas de buscarlo, puede encon- trar otros ítemes interesantes mientras busca el deseado, o puede perder el interés por el ítem original frente a otras alternativas que Llaman su atención. Es decir, el comportamiento de browsing no es algo exclusivo de las búsquedas vagas o mal definidas, sino que es algo consustancial a la búsqueda humana de información. Por tanto, proporcionar métodos para un browsing abierto y exploratorio de la base de datos, esto es, que llegue más allá del clásico y limitado examen lineal de las referencias recuperadas o del vocabulario de indización, se convierte en un requisito imprescindible para cualquier OPAC.

Para que el catálogo en línea ofrezca estas prestaciones es necesario que su base de datos contenga una gran diversidad de tipos de información (texto completo, sumarios, índices, listas de obras citadas, etcétera) y que esté organizada de manera que permita un fácil movimiento entre ellos. De esta forma, sería posible llevar a cabo en un catálogo en Línea todas esas numerosas y variadas operaciones que se efectúan cuando se busca información en un contexto manual: a partir de una obra o autor conocido se buscan otras similares con el mismo encabezamiento de ma- teria o número de clasificación, se usa la lista de obras citadas en un documento para encontrar otras de interés, se miran las tablas de los esquemas de clasificación para ver cuál es el número que corresponde a temas más genéricos o más específicos que aquella obra de la que sabemos su número de clasificación, se hojea el texto o índice de aquellas obras situadas juntas en los estantes de la biblioteca, etcétera.

La consecución de estos objetivos requiere, por tanto, no sólo incluir en la base de datos todo tipo de información, sino también organizarla de manera que sea sencillo moverse de uno a otro. A este respecto, el hipertexto parece ser la opción que mejores prestaciones ofrece, aunque presenta importantes problemas de desorientación en bases de datos de gran tamaño, que deberán resolverse con alguno de los métodos ya mencionados previamente: browser gráfico, estructura de dos niveles mediante el uso combinado de técnicas de clustering y esquemas de clasificación, etcétera.

\section{REFERENCIAS BIBLIOGRÁFICAS}

AKEROYD, J. (1990). "Information seeking in online catalogues". Journal of Documentation, 46(1), 33-52.

APTED, S. M. (1971). "General purposive browsing". Library Association Record, 73(12), 228-230.

BAKER, S. L. (1986). "Overload, browsers and selections". Library and Information Science Research, 8(4), 315-329.

BATES, M. J. (1979). "Idea tactics". Journal of the American Society for Information Science, 30(5), 280-289.

—. (1989). "The design of browsing and berrypicking techniques for the online search interface". Online Review, 13(5), 407-424.

BAWDEN, D. (1986). "Information systems and the stimulation of creativity". Journal of Information Science, 12(5), 203-216.

—. (1993). "Browsing: theory and practice". Perspectives in Information Management, 3(1), 71-85.

BEHESHTI, J. (1992). "Browsing through public acces catalogs". Information Technology and Libraries, 11(3), 220-228.

BELKIN, N. J.; P. G. Marchetti; C. Cool (1993). "BRAQUE: design of an interface to support user interaction in information retrieval". Information Processing and Management, 29(3), 325-344.

BENEST, I. D.; G. Morgan; M. D. Smithurst (1987). "A humanised interface to an electronic library". INTERACT'87: proceedings of the 2nd IFIP'87 International Conference on Human-Computer Interaction (Stuttgart, 1-14 September 1987). Amsterdam: North-Holland, 905-910. 
BORGMAN, C. L. (1989). “All users of information retrieval systems are not created equal: an exploration into individual differences". Information Processing and Management, 25(3), 237-251.

—.et al. (1995). "Children's searching behavior on browsing and keyword online catalogs: the Science Library Catalog Project”. Journal of the American Society for Information Science, 46(9), 663-684.

CHANG, S. J.; R.E. Rice (1993). "Browsing: a multidimensional framework". Annual Review of Information Science and Technology, 28, 231-275.

COVE, J. E; B.C. Walsh (1988). "Online text retrieval via browsing". Information Processing and Management, 24(1), 31-37.

CROFT, W. B.; R. H. Thompson (1987). " $1{ }^{3}$ R: a new approach to the design of document retrieval systems". Journal of the American Society for Information Science, 38(6), 389-404.

DAVIES, R. (1989). "The creation of new knowledge by information retrieval and classification". Journal of Documentation, 45(4), 273-301.

DERVIN, B.; M. Nilan (1986). "Information needs and uses". Annual Review of Information Science and Technology, 21, 3-33.

DILLON, M. (1992). "The graphical browse project". Annual Review of OCLC Research, July 1991-June 1992, 22-25.

EDWARDS, D. M.; L. Hardman (1989). “'Lost in hyperspace’: cognitive mapping and navigation in a hypertext environment". McAleese, R., ed. Hypertext: theory into practice. Norwood, NJ: Ablex, 105-125.

ELLIS, D. (1989). "A behavioral approach to information retrieval system design”. Journal of Documentation, 45(3), 171-212.

FOSS, C. L. (1989). "Tools for reading and browsing hypertext”. Information Processing and Management, 25(4), 407-418.

FREEMAN, R. R.; P. Atherton (1968). "File organization and search strategy using the Universal Decimal Classification in mechanized reference retrieval systems". Mechanized information storage, retrieval and dissemination: proceedings of the FID/IFIP joint conference. Amsterdam: North-Holland, 122-152.

HANCOCK, M. M. (1987). "Subject searching behaviour at the library catalogue and at the shelves: implications for online interactive catalogues". Journal of Documentation, 43(4), 303-321.

HERNER, S. (1970). Browsing. En: Kent, A. y Lancour, H., eds. Encyclopedia of library and information science, 3, 408-415.

HILDRETH, C. R. (1982). "The concept and mechanics of browsing in an online library catalog". Proceedings of the 3rd National Online Meeting (New York, March 30-April 1 1982). Medford, NJ: Learned Information, 181-196.

—. (1989). "General introduction; OPAC research: laying the groundwork for future OPAC design". En: Hildreth, C. R., ed. The online catalog: developments and directions. London: Library Association, 1-24.

HJERPPE, R. (1986). "Project HYPERCATaIog: visions and preliminary conceptions of an extended and enhanced catalog". En: Brookes, B. C., ed. Intelligent information systentr for the information society. Amsterdam: North-Holland, 211-232.

—. (1989). "HYPERCAT at LIBLAB in Sweden: a progress report". En: Hildreth, C. R., ed. The online catalog: developments and directions. London: Library Association, 177-209.

KUHLTHAU, C. C. (1991). "Inside the search process: information seeking from the user's perspective". Journal of the American Society for Information Science, 42(5), 361-371.

KWASNIK, B. H. (1992). "The functional components of browsing”. Annual Review of OCLC Research, July 1991-June 1992, 53-56.

LARSON, R. R. (1991). “Classification clustering: probabilistic information retrieval and the online catalog”. Library Quarterly, 61(1), 133-173.

—. et al. (1996). "Cheshire II: designing a next-generation online catalog". Journal of the American Society for Information Science, 47(7), 555-567. 
LIU, S.; E. Svenonius (1991). "DORS: DDC online retrieval system”. Library Resources and Technical Services, 35(4), 359-375.

MORSE, P. M. (1970). "Search theory and browsing”. Library Quarterly, 40(4), 391-408.

NOERR, P. L.; K. T. Bivins-Nֶoerr. (1985). "Browse and navigate: an advance in database access methods. Information Processing and Management, 21(3), 205-213.

NORTON, T. (1984). "Secondary publications have future in libraries". Aslib Proceedings, 36(5), 317-323.

O'CONNOR, B. (1988). "Fostering creativity: enhancing the browsing environment". International Journal of Information Management, 8(3), 203-210.

ODDY, R. N. (1977). "Information retrieval through man-machine dialogue". Journal of Documentation, 33(1), 1-14.

PALAY, A. J.; M. S. Fox (1981). "Browsing through databases". En: Oddy, R. N. et al., eds. Information retrieval research. London: Butterworth, 310-324.

POLLARD, R. (1993). "A hypertext-based thesaurus as a subject browsing aid for bibliographic databases". Information Processing and Management, 29(3), 345-357.

ROSENBERG, J. B.; C. L. Borgman (1992). "Extending the Dewey Decimal Classification via keyword clustering: the Science Library Catalog Project”. ASIS' 92: proceedings of the 55th ASIS annual meeting (Pittsburgh, October 26-29 1992). Medford, NJ: Learned Information, 171-184.

SARACEVIC, T.; P. Kantor (1988). "A study of information seeking and retrieving, III. Searchers, searches, and overlap”. Journal of the American Society for Information Science, 39(3), 197-216.

TURTLE, H. R.; W. B. Croft (1991). "Evaluation of an inference network-based retrieval model”. ACM Transactions on Information Systems, 9(3), 187-222.

URQUHART, D. J. (1976). "National lending/reference libraries or libraries of first resort". BLL Review, 4(1), 7-10.

WADE, S. J.; P. Willett (1988). "INSTRUCT: a teaching package for experimental methods in information retrieval. Part III. Browsing, clustering and query expansion". Program, 22(1), 44-61.

—. P. Willett; D. Bawden. (1989). "SIBRIS: the Sandwich interactive browsing and ranking information system". Journal of Information Science, 15(4/5), 249-260.

\section{GLOSARIO}

Berrypicking.- búsqueda evolutiva. Modelo de recuperación de información que describe la búsqueda como un proceso de aceptación y/o rechazo de información/documentos según unos parámetros que se van modificando a medida que se desarrolla la búsqueda.

Idea tactics.- tácticas para generar ideas. En la búsqueda de información, métodos que ayudan a crear nuevas ideas que ayuden a perfilar la estrategia.

Area scanning.- examen de área. En la búsqueda de información, técnica consistente en ojear los materiales impresos situados en un área (física o del conocimiento) donde con anterioridad se ha encontrado material de interés.

Feedback.- reacción, interacción. En la búsqueda de información, reacción del usuario ante la información que el sistema le presenta lo que permite que este proceso sea interactivo.

Browsable.- consultable, ojeable, hojeable. En la búsqueda de información, entorno o contexto que permite la consulta de un modo aleatorio como opuesto a una búsqueda de información concreta y exacta.

Kwic.- índice que contiene los títulos ordenados alfabética-mente por cada una de las palabras con contenido y presenta-dos de modo que la palabra clave aparece en el centro de la pantalla con el resto del título a ambos lados en su orden.

Library map.- mapa de la biblioteca. Representación virtual del edificio de la biblioteca y su contenido.

Best match model.- modelo de recuperación de información por coincidencia óptima. Técnica usada en la recuperación de información por la que se utiliza la información del usuario sobre la pertinencia de los documentos recuperados para volver a formularla.

Clustering.- agrupamiento. Formación de conjuntos de documentos, referencias, etcétera, por alguna característica común, como puede ser el tema.

Browsing room.- salón de lectura. En una biblioteca, sala de lectura de ambiente relajado, libre de algunas de las normas bibliotecarias y especialmente dedicada a la promoción de la lectura.

Browser.- interfaz gráficos de consulta imprecisa. En la búsqueda de información, diagrama esquemático hipertextual que muestra al usuario las dependencias y fondos de la biblioteca. 